\title{
Cosmic Censorship in Quantum Einstein Gravity
}

\author{
A. Bonanno \\ INAF, Osservatorio Astrofisico di Catania, via S. Sofia 78, I-95123 Catania, Italy and \\ INFN, Sezione di Catania, via S. Sofia 64, I-95123, Catania, Italy. \\ B. Koch \\ Instituto de Física, Pontificia Universidad Católica de Chile, Av. Vicuna Mackenna 4860, Santiago, Chile \\ A. Platania \\ INAF, Osservatorio Astrofisico di Catania, via S. Sofia 78, I-95123 Catania, Italy \\ INFN, Sezione di Catania, via S. Sofia 64, I-95123, Catania, Italy. and \\ Università di Catania, via S. Sofia 63, I-95123 Catania, Italy
}

\begin{abstract}
We study the quantum gravity modification of the Kuroda-Papapetrou model induced by the running of the Newton's constant at high energy in Quantum Einstein Gravity. We argue that although the antiscreening character of the gravitational interaction favours the formation of a naked singularity, quantum gravity effects turn the classical singularity into a "whimper" singularity which remains naked for a finite amount of advanced time.
\end{abstract}

PACS numbers: 04.20.Dw, 11.10.Hi, 04.60.-m

\section{INTRODUCTION}

Spacetime singularities signal the breakdown of the classical description within General Relativity. Such singularities typically appear at the beginning of the universe and at the endpoint of a gravitational collapse. In the latter case, after the star's irregularities have been radiated away and the external field relaxes to its Kerr-Newman geometry, the structure of the interior solution is rather uncertain, even classically. According to the still unproven cosmic censorship hypothesis the singularity at $r=0$ in a physical black hole ought to be spacelike and its "evolution" is described by a generic mixmaster solution. In particular it is believed that when the curvature reaches Planckian values quantum fluctuations of the geometry have a self-regulator effect and can eventually halt the collapse.

An interesting possibility, already proposed in the context of minisuperspace models [1, 2] coupled to matter fields, is a transition to a de Sitter core of size $\hbar G \equiv l_{\mathrm{pl}}$ [3, 4, a scenario recently emerged also in the framework of Asymptotically Safe (AS) gravity [5, 6] and loop quantum gravity [7, 8. On the other hand, the dynamical role of quantum fluctuations of the metric in the formation of the event horizon (EH) and of the central singularity is still unknown.

In contrast to the cosmological singularity, a black hole singularity at $r=0$ develops in time because the radial coordinate is time-like inside of the black hole $(\mathrm{BH})$ nucleus. The classical evolution is essentially determined by two effects: the radiation from the collapsing star, and the backscattered inflow of gravitational waves which takes the form $v^{-4 l-4}$, where the advanced time $v \rightarrow \infty$ and $l$ is the spherical harmonic multipole of the perturbation [9]. The latter is dominant in the interior only at large advanced times because any perturbation of the metric $\delta g$ will decay as $v^{-2 l-2}$ near $r=0$ [10]. The details of the collapse are therefore essential for the understanding the structure of the singularity at early advanced time, at least for a spherical BH with small asphericities.

In this work we study possible quantum gravity modifications occurring at the early part of the singularity, by linking the dynamics of the collapse with the structure of the singularity in an quantum-corrected BH interior. An attempt to answer to the above issue can be pursued within the framework of AS gravity [11 13], in particular in the case of Quantum Einstein Gravity (QEG) [14-16].

The basic input of this approach is the existence of a Non-Gaussian Fixed Point (NGFP) of the Renormalization Group (RG) flow of the gravitational interaction that allows the definition of a new set of relevant operators with a well behaved ultraviolet (UV) limit. In QEG the antiscreening character of the gravitational constant at high energies has a physically clear interpretation in terms of "paramagnetic dominance" of the vacuum [17, and it is possible to encode the running of $G$ in a self-consistent manner, by renormalization group improving the Einstein equations, as explained in cosmological contexts [18, 22 .

Recent studies have modeled the collapse in terms of an homogeneous interior surrounded by a Renormalization Group (RG) improved Schwarzschild exterior [23, 24. In this work we instead focus on the dynamical process of $\mathrm{BH}$ formation and discuss the structure of the singularity in a quantum gravity corrected Kuroda-Papapetrou (KP) model 25, 26. We shall show that a violation of the Cosmic Censorship conjecture is in general favoured by quantum gravity, although the final singularity at $r=0$ turns out to be a rather weak, "whimper" singularity, according to the 
the Tipler classification [27]. We argue that, at least in spherical symmetry our result should be a generic outcome of the gravitational collapse in QEG.

The rest of this paper is organized as follows. In section II we introduce the basic ideas and we find a RG improved metric describing the gravitational collapse within the VKP model. The general solution of the geodesic equation is studied in the section III, while in section IV we focus on the analysis of the "nature" of the singularity in comparison to the classical case. Finally, in section $\mathrm{V}$ we summarize our main results and comment on possible extensions and applications.

\section{BASIC EQUATIONS}

In the framework of the RG flow for gravity, the "emerging" geometry at the energy scale $k$ can be obtained by solving the RG-improved Einstein's equations

$$
R_{\mu \nu}\left[\langle g\rangle_{k}\right]-\frac{1}{2} R\left\langle g_{\mu \nu}\right\rangle_{k}=\Lambda(k)\left\langle g_{\mu \nu}\right\rangle_{k}+8 \pi G(k)\left\langle T_{\mu \nu}\right\rangle_{k}
$$

which can be derived from the effective average action for gravity $\Gamma_{k}\left[g_{\mu \nu}\right]$ via

$$
\frac{\delta \Gamma_{k}}{\delta g_{\mu \nu}(x)}\left[\langle g\rangle_{k}\right]=0 \text {. }
$$

Here, $\Gamma_{k}\left[g_{\mu \nu}\right]$ is a free energy functional which depends on the metric and a momentum scale $k$ which is usually considered as an infrared cutoff. This functional is similar to the ordinary effective action which is reproduced in the $k \rightarrow 0$ limit. As the functional $\Gamma_{k}$ gives rise to an effective field theory valid near the scale $k$, when evaluated at tree level it describes all quantum gravitational phenomena at tipical momentum $k$. In the case of QEG, the functions $G(k)$ and $\Lambda(k)$ are computed from the exact flow equation for gravity 28] from the ansatz,

$$
\Gamma_{k}[g]=(16 \pi G(k))^{-1} \int \sqrt{g}\{-R(g)+2 \Lambda(k)\} d^{4} x .
$$

Clearly, the dynamical implications of a running Newton's constant can only be fully exploited once a prescription $k \rightarrow k\left(x^{\mu}\right)$ is provided to close the system (1).

In the case of pure gravity it has been argued that the correct cutoff identification is provided by the proper distance of a free-falling observer radially falling into a $\mathrm{BH}$ (at least in spherical geometry [5]). On the other hand if matter is present in the system the ultraviolet structure of the renormalized flow can be strongly deformed at high temperatures. It is known from scalar field theory [29, 30] that the renormalization scale $k$ and the temperature $T$ play, up to logarithmic corrections, a similar role in determining the scaling laws so that $k \sim T$.

In the case at hand, as the $\mathrm{BH}$ interior is homogeneous, it is natural to assume that near the singularity the matter can be described by an equation of state consisting of a fluid with radiation type energy density $\rho$, so that

$$
\rho \sim T^{4} \sim k^{4}
$$

This relation can then be inverted and, for actual calculation, we can write $k=\xi \sqrt[4]{\rho}$ where $\xi$ is a positive constant whose precise value does not qualitatively change the conclusions of our discussion, as we shall see. It is important to remark that this is the only possible choice compatible with a conformally invariant theory at the NGFP, as gravity is supposed to be. The introduction of a generic functional form of the type $k=k(\rho)$ would imply the presence of other mass scales not allowed at the NGFP.

In order to solve (1) it is necessary to resort to some approximation. As discussed in [5] a first step is to construct a self-consistent solution which interpolates from the classical one expected at infra-red scales $k \rightarrow 0$ and the full quantum corrected geometry expected at ultra-violet scales $k \rightarrow \infty$. Our strategy in this work therefore amounts to use an iterative procedure: we shall first RG-improve the classical Vaidya solution with the cutoff identification (4) and then compute the new quantum corrected stress-energy tensor following (1).

Let us write the (average) metric of a spherically symmetric collapsing object as a generalized Vaidya metric 31 . of the type

$$
\left\langle d s^{2}\right\rangle_{k}=-f_{k}(r, v) d v^{2}+2 d v d r+r^{2} d \Omega^{2},
$$

where $f_{k}(r, v)=1-2 M_{k}(r, v) / r$ is the RG-improved lapse function. The non-zero components of the Einstein tensor read

$$
G_{v r}=-\frac{2}{r^{2}} \frac{\partial M_{k}(r, v)}{\partial r}, \quad G_{v v}=\frac{2}{r^{2}} \frac{\partial M_{k}(r, v)}{\partial v}+\frac{2 f_{k}(r, v)}{r^{2}} \frac{\partial M_{k}(r, v)}{\partial r}
$$




$$
G_{\theta \theta}=r \frac{\partial^{2} M_{k}(r, v)}{\partial r^{2}}, \quad G_{\phi \phi}=r \frac{\partial^{2} M_{k}(r, v)}{\partial r^{2}}(\sin \theta)^{2} .
$$

As discussed in [32, 33] the energy-momentum tensor has a null-part and non-null part so that

$$
T_{\mu \nu}=\rho l_{\mu} l_{\nu}+(\sigma+p)\left(l_{\mu} n_{\nu}+l_{\nu} n_{\nu}\right)+p g_{\mu \nu}
$$

where $n_{\mu} l^{\mu}=-1, l_{\mu} l^{\nu}=0$ and

$$
\rho(r, v)=\frac{1}{4 \pi G_{0} r^{2}} \frac{\partial M_{k}(r, v)}{\partial v}
$$

and

$$
\sigma(r, v)=\frac{1}{4 \pi G_{0} r^{2}} \frac{\partial M_{k}(r, v)}{\partial r}, \quad p(r, v)=-\frac{1}{8 \pi G_{0} r} \frac{\partial^{2} M_{k}(r, v)}{\partial r^{2}} .
$$

Classically $M_{0}(r, v)=G_{0} m(v)$, being $G_{0}$ the usual Newton's constant and $m(v)$ a dynamical mass function which is a function of the advanced time $v$. In this case, as first discussed in [34, 35, the stress energy tensor is that of a null fluid and the classical field equations simply read $\dot{m}(v) / 4 \pi r^{2}=\rho(r, v)$. In the iterative RG-improvement approach the energy density is computed at the bare (classical) level and the quantum corrected lapse function is then obtained via the substitution

$$
G_{0} \rightarrow G(k) \equiv \frac{G_{0}}{1+\omega G_{0} k^{2}}, \quad M_{0}(r, v) \rightarrow M_{k}(r, v)=G(k) m(v),
$$

where $\omega=1 / g_{*} \approx 1.5, g_{*}$ is the value of the dimensionless running Newton's constant at the NGFP' and $k$ is the infrared cutoff defining the running function $G(k)$ (note that $G(0) \equiv G_{0}$ ). The scaling of the Newton's constant as a function of the energy scale $k$ given in eq. 111 is obtained by solving the beta functions within the Asympototic Safety Scenario for Quantum Gravity [5].

As we discussed before, $k=k(r, v)$ can be written in terms of the energy density of the ingoing radiation via an equation of state for pure radiation. Let us assume that the ingoing flow of radiation is injected in the spacetime at $v=0$ and then stopped at $v=\bar{v}$. The mass of the collapsing object thus increases until at $v=\bar{v}$ the accretion stops, and it can be written as

$$
m(v)= \begin{cases}0 & v<0 \\ \lambda v & 0 \leq v<\bar{v} \\ \bar{m} & v \geq \bar{v}\end{cases}
$$

The description of the classical gravitational collapse throught the mass function given in (12) constitutes the VaidyaKuroda-Papapetrou (VKP) model [25, 26, 35].

In the RG improved model, for $0 \leq v<\bar{v}$ the lapse function has the following form

$$
f_{k}(r, v)=1-\frac{2 \lambda G_{0} v}{r+\alpha \sqrt{\lambda}}, \quad \text { with } \quad \alpha=\frac{\xi^{2} G_{0}}{\sqrt{4 \pi} g_{*}}
$$

while for $v \geq \bar{v}$ it continuosly matchs with a quantum corrected Schwarzschild spacetime with

$$
f_{k}(r)=1-\frac{2 \bar{m} G_{0}}{r+\alpha \sqrt{\lambda}} .
$$

Therefore the RG-improvement gives rise to a generalized Vaidya-Kuroda-Papapetrou (VKP) model whose mass function reads

$$
M_{k}(r, v)=\frac{G_{0} r}{r+\alpha \sqrt{\lambda}} m(v)
$$

\footnotetext{
1 The existence and the positivity of the NGFP depend in general on the matter content of the system [36]. In our iterative approach we use the expression of $G(k)$ obtained from RG methods within the Einstein-Hilbert truncation, and neglect any possible modification coming from the addition of matter fields.
} 
where the mass $m(v)$ is the one of the classical VKP-model, given eq. 12. As expected, the continuity of the RGimproved mass function $M_{k}(r, v)$ along the $v=\bar{v}$ light-cone implies that the quantum corrected spacetime doesn't converge to "pure" Schwarzschild, but only approaches it asymptotically (for $r \rightarrow \infty$ ). The energy momentum tensor for $v \geq \bar{v}$ is characterized by an effective energy density and pressure given by

$$
\sigma(r)=\frac{\lambda \bar{v}}{4 \pi r^{2}} \frac{\alpha \sqrt{\lambda}}{(r+\alpha \sqrt{\lambda})^{2}}, \quad p(r)=\frac{\lambda \bar{v}}{4 \pi r} \frac{\alpha \sqrt{\lambda}}{(r+\alpha \sqrt{\lambda})^{3}}
$$

respectively. A similar situation is encountered in the RG-improved Schwarzschild solution of [5], where the RGimproved Schwarzshild solution is also not a solution of vacuum Einstein equation.

From equation (13) it is clear that if $\alpha \neq 0$ (for $\alpha=\xi=0, f_{k}$ reduces to the classical lapse function) the improved lapse function $f_{k}(r, v)$ is well defined in the limit $r \rightarrow 0$

$$
\lim _{r \rightarrow 0} f_{k}(r, v)=1-\frac{\sqrt{16 \pi \lambda}}{\omega \xi^{2}} v
$$

and thus the metric is regular in $r=0$. We have to remark that although the metric is regular at $r=0$, this hypersurface is actually singular. The Ricci curvature $R$ and the Kretschmann scalar $K=R_{\alpha \beta \gamma \delta} R^{\alpha \beta \gamma \delta}$ are divergent in $r=0$, in particular

$$
R=-\frac{G_{0} \sqrt{\lambda} v}{\alpha r^{2}}+O(1 / r), \quad K=\frac{16 G_{0} \sqrt{\lambda} v}{\alpha^{2} r^{4}}+O\left(1 / r^{3}\right) .
$$

Nevertheless, it should be noticed that the $r \rightarrow 0$ behavior is less singular than in the classical case, for which $K \sim 1 / r^{6}$.

The zeros of the lapse function $f_{k}(r, v)$ identify the so called apparent horizons (AH). In the classical case the apparent horizon is described by the equation $r_{\mathrm{AH}}(v)=2 m(v) G_{0}$, while for the RG improved Vaidya metric it is shifted by the constant $\alpha \sqrt{\lambda}$

$$
r_{\mathrm{AH}}(v)=2 m(v) G_{0}-\alpha \sqrt{\lambda}=2 m(v) G_{0}-\frac{G_{0} \xi^{2}}{g_{*}} \sqrt{\frac{\lambda}{4 \pi}},
$$

with the condition $r_{\mathrm{AH}}(v) \geq 0$. At the end of the process the apparent and event horizons (discussed in the next section) must converge to the improved Schwarzchild radius $r_{S}=2 \bar{m} G_{0}-\alpha \sqrt{\lambda}$. The final mass $\bar{m}$ of the BH, the value of $\bar{v}$, and the value of $\lambda$ are thus related by the condition $\bar{m}=\lambda \bar{v}$. Furthermore, since the Schwarzchild radius $r_{S}$ must be positive, there exists a minimum period $\bar{v}$ of irradiation, necessary to form a black hole

$$
r_{S}=2 \lambda \bar{v} G_{0}-\alpha \sqrt{\lambda} \geq 0 \quad \Rightarrow \quad \bar{v} \geq v_{\min }(\lambda) \equiv \frac{\xi^{2}}{2 g_{*}} \sqrt{\frac{1}{4 \pi \lambda}}
$$

Here, the minimum value $v_{\text {min }}$ is a function of the radiation rate $\lambda$.

\section{OUTGOING RADIAL NULL GEODESICS}

\section{A. Classical VKP model}

It is useful to remind the basic properties of the classical VKP model [37, 38]. In the Vaidya geometry outgoing radial light rays are represented as solutions of

$$
\frac{d r}{d v}=\frac{1}{2}\left(1-\frac{2 G_{0} m(v)}{r}\right) .
$$

If $m(v)=\lambda v$ the evolution of the spacetime is halted at $r=0$ by a strong spacelike singularity [25], as it will be discussed in the next section. In particular it is possible to show that if $\lambda>\lambda_{c}=\frac{1}{16 G_{0}}$ the singularity in $r=0$ is always covered by an event horizon. On the other hand, if $\lambda \leq \lambda_{c}$ two linear solutions of eq. (21), $r_{ \pm}(v)=\mu_{ \pm} v$ with

$$
\mu_{ \pm}=\frac{1 \pm \sqrt{1-16 \lambda G_{0}}}{4}
$$



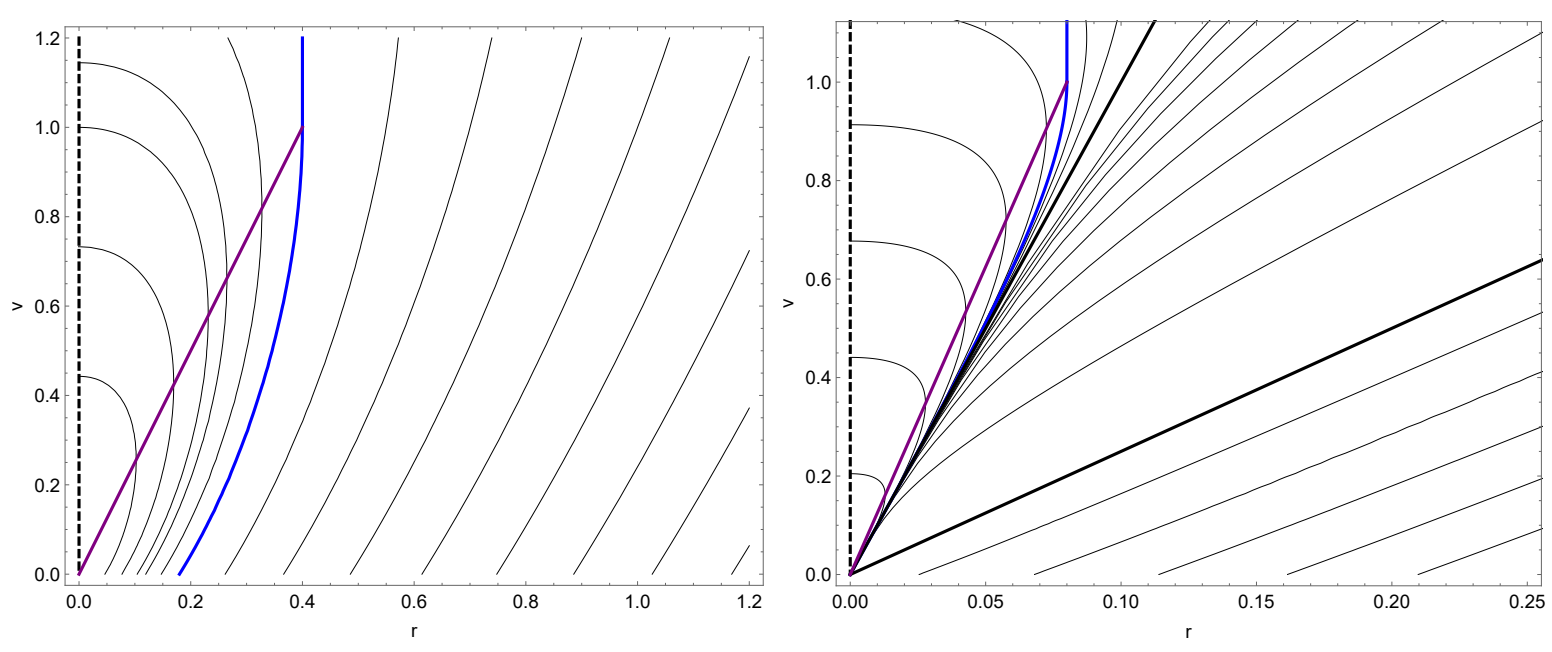

FIG. 1. Phase diagram $(r(v), v)$ in the classical VKP model. The blue line is the event horizon (EH), the purple line is the apparent horizon $(\mathrm{AH})$, and the black lines represent the family of solutions of the classical geodesic equation. Left panel: For $\lambda>\frac{1}{16 G_{0}}$ the singularity in $r=0$ is covered by the event horizon. Right panel: In the case $\lambda \leq \frac{1}{16 G_{0}}$ a Cauchy horizon $r_{+}(v)=\mu_{+} v$ (external bold black line) is formed, and the singularity is naked.

appear. The positive one is the Cauchy horizon, the other one is the tangent (in the $(r(v), v)$ space) to the event horizon at the "point" $(r=0, v=0)$. In this case the singularity is globally naked: all the solutions belonging to the region between the two linear solutions represent light rays that start from the singularity $(r=0, v=0)$ and will reach the observer at infinity. In particular the Cauchy horizon is the first light ray escaping from the singularity.

The general solution to 21 reads

$$
-\frac{2 \operatorname{ArcTan}\left[\frac{v-4 r(v)}{v \sqrt{-1+16 \lambda G_{0}}}\right]}{\sqrt{-1+16 \lambda G_{0}}}+2 \log (v)+\log \left[2 \lambda G_{0}-\frac{r(v)}{v}+2 \frac{r^{2}(v)}{v^{2}}\right]=C
$$

where $C$ is an arbitrary integration constant. A set of this family of solutions, obtained by varying the constant $C$, is shown in fig. (1). One finds that the family of solutions of (21) for $\lambda \leq \lambda_{c}$ can be described by the following implicit equation

$$
\frac{\left|r(v)-\mu_{-} v\right|^{\mu_{-}}}{\left|r(v)-\mu_{+} v\right|^{\mu_{+}}}=\tilde{C}
$$

where $\tilde{C}$ in an arbitrary complex constant [39]. Note that the linear solutions $r_{ \pm}(v)$ are recovered as particular cases of this implicit equation.

\section{B. RG-improved VKP model}

If we now encode the leading quantum correction to this model by using 13 to form the new Vaidya line element, the equation for the outgoing radial null geodesic reads

$$
\dot{r}(v)=\frac{1}{2}\left(1-\frac{2 \lambda v G_{0}}{r(v)+\alpha \sqrt{\lambda}}\right),
$$

where it can be noticed that the effect of a running gravitational coupling is to shift $r(v)$ by a factor $\alpha \sqrt{\lambda}$. In particular, if $\lambda \leq \frac{1}{16 G_{0}}$ the solutions of 25 are implicitly defined by

$$
\frac{\left|r(v)+\alpha \sqrt{\lambda}-\mu_{-} v\right|^{\mu_{-}}}{\left|r(v)+\alpha \sqrt{\lambda}-\mu_{+} v\right|^{\mu_{+}}}=\tilde{C}
$$

and the two linear solutions are now given by

$$
r_{ \pm}(v)=-\alpha \sqrt{\lambda}+\mu_{ \pm} v
$$




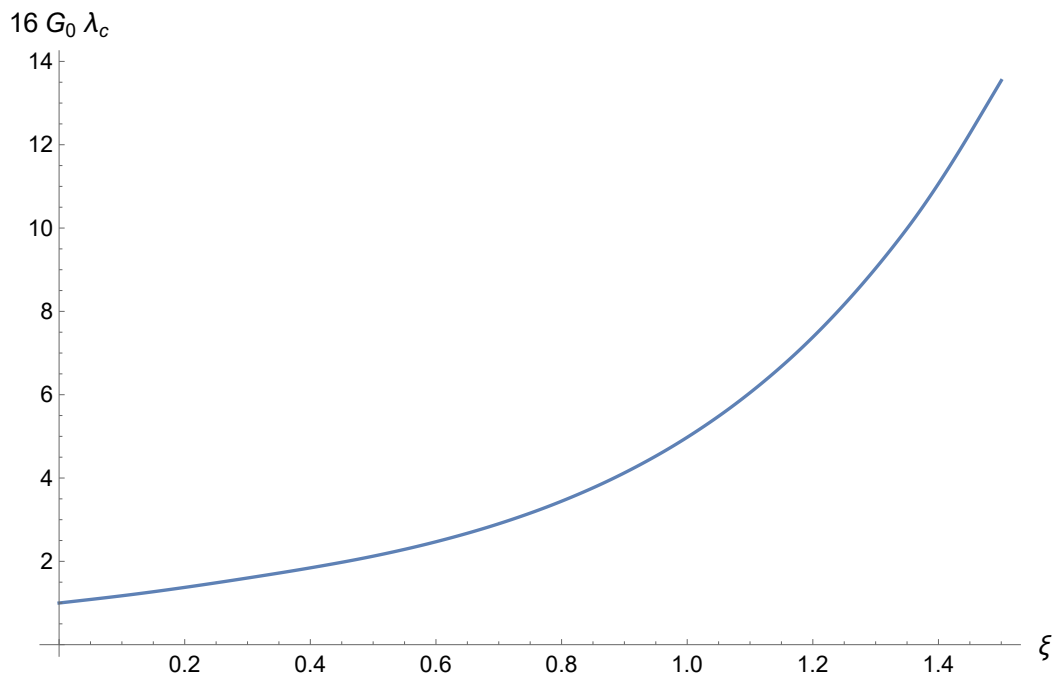

FIG. 2. Relative critical value $\lambda_{c} / \frac{1}{16 G_{0}}$ as a function of $\xi$. As it is clear from the picture, $\lambda_{c}$ increases monotonically with $\xi$ and in particular it reduces to the classical value when $\xi=0$.
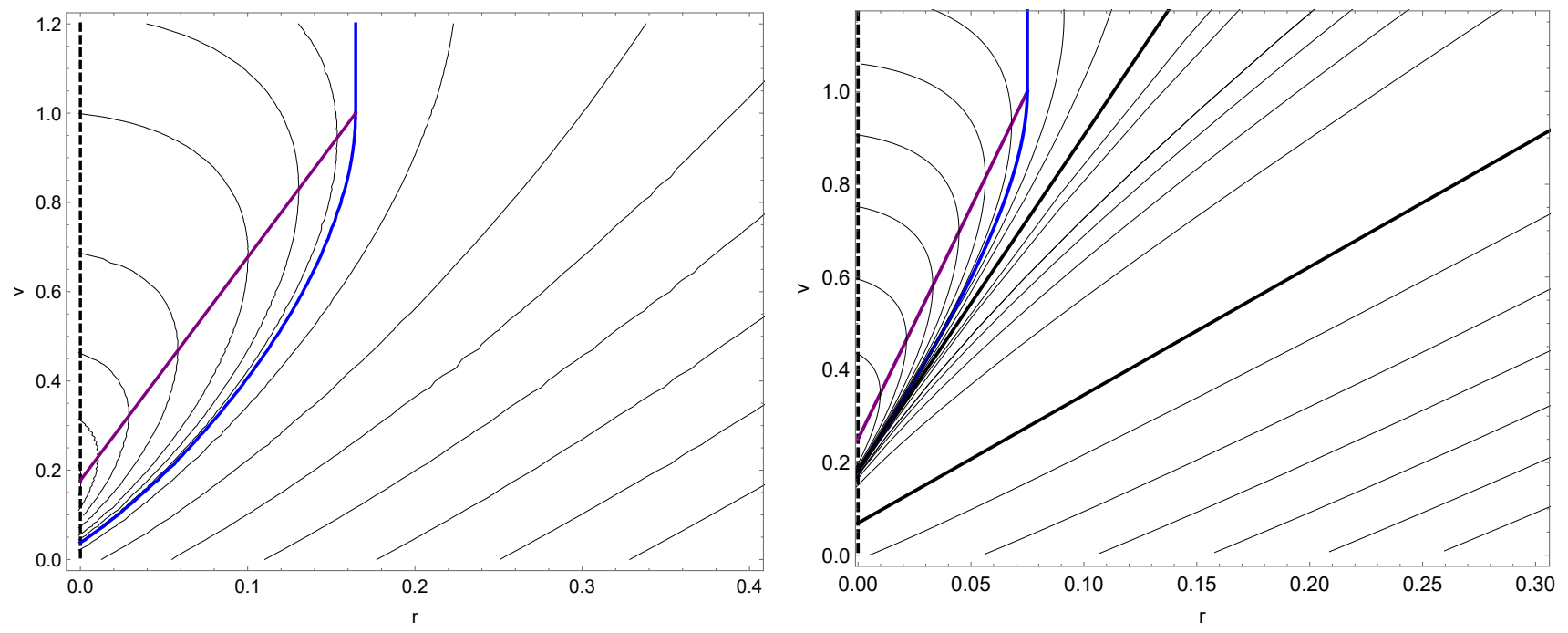

FIG. 3. Phase diagram $(r(v), v)$ in the improved VKP model, for $\frac{1}{16 G_{0}}<\lambda \leq \lambda_{c}$ (left panel) and $\lambda_{c} \leq \frac{1}{16 G_{0}}$ (right panel). The blue line is the $\mathrm{EH}$, the purple line is the $\mathrm{AH}$, and the black curves are particular solutions of the improved geodesic equation. For $\lambda \leq \lambda_{c}$ the singularity in $r=0$ is globally naked, as the EH forms just after the formation of the singularity. Since in the improved case the affine solution $r_{+}(v)$ has no longer the meaning of a Cauchy horizon, the two cases depicted in these pictures are physically the same.

with $\mu_{ \pm}$defined in 22 . These solutions are obtained only for $\lambda \leq \frac{1}{16 G_{0}}$ but, in contrast to the classical case, the value $\lambda_{c}$ at which the singularity become globally naked is now greater than $\frac{1}{16 G_{0}}$. Furthermore, the solution $r_{+}(v)$ in eq. 27) has no longer the meaning of Cauchy horizon, since it emerges from $r=0$ well after the formation of the singularity, i.e. for $v>0$.

In this case it is not possible to determine analytically the critical value $\lambda_{c}$ but one has to determine it numerically as the value of $\lambda$ such that the radius of the outgoing null geodesic is zero at $v=0$. It turns out that $\lambda_{c}$ is always greater than its classical counterpart, and in particular the dependence of the critical value $\lambda_{c}$ on the RG parameter $\xi$ is reported in fig. 2.

By analyzing the solutions of the geodesic equation, we studied the general behavior of the trajectories in the phase diagram depending on the value of the radiation rate $\lambda$. As it can be seen in the fig. 3, for $\lambda \leq \lambda_{c}$ the singularity is globally naked and moreover it extends in time, up to the formation of the event horizon for $v>0$. Moreover, as $r,{ }_{\mu} r^{, \mu}=f_{k}(r, v)$, the singularity $r=0$ is timelike for $v<\tilde{v}$, null for $v=\tilde{v}$ and spacelike for $v>\tilde{v}$, where $\tilde{v}=\frac{\alpha \sqrt{\lambda}}{2 \lambda G_{0}}$ 


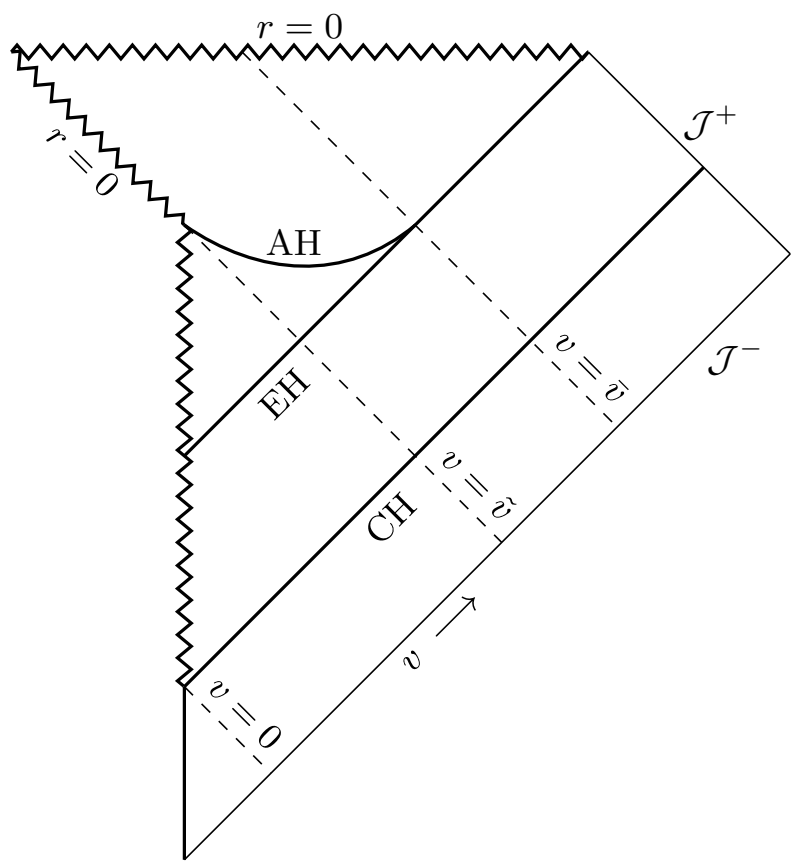

FIG. 4. Global structure of the spacetime for $\lambda \leq \lambda_{c}$.

is the value of $v$ at which the improved $\mathrm{AH}$ forms, $r_{\mathrm{AH}}(\tilde{v})=0$. The global structure of the spacetime is described in fig. 4. We can conclude that at least within the VKP model, quantum gravity effects can favor the presence of naked singularities. However, as it will be discussed in the next section, the global effect of a running Newton's constant is to render the singularity in $r=0$ much milder and integrable.

\section{NATURE OF THE SINGULARITY IN THE IMPROVED VAIDYA SPACE-TIME}

As it was already noticed, the divergence of Ricci curvature $R$ and the Kretschmann scalar $K$ is different in the case of the Vaidya improved metric. Let us discuss this issue in detail by following the approach of [32. We write the geodesic equation for null rays of the line element (5) as a dynamical system

$$
\left\{\begin{array}{l}
\frac{\mathrm{d} v(t)}{\mathrm{d} t}=N(r, v) \\
\frac{\mathrm{d} r(t)}{\mathrm{d} t}=D(r, v)
\end{array}\right.
$$

where $t$ is a parameter and the functions $N(r, v)$ and $D(r, v)$ are defined as

$$
N(r, v)=2 r \quad D(r, v)=r-2 M_{k}(r, v) .
$$

The singularities are the fixed point solutions of the system (28) (i.e. $r=0$ and $M_{k}(0, v)=0$ ). The behavior of the trajectories near the singularity can be studied by linearizing the system around the fixed point solution

$$
\left\{\begin{array}{l}
\frac{\mathrm{d} v(t)}{\mathrm{d} t}=\dot{N}_{\mathrm{FP}}\left(v-v_{\mathrm{FP}}\right)+N_{\mathrm{FP}}^{\prime}\left(r-r_{\mathrm{FP}}\right) \\
\frac{\mathrm{d} r(t)}{\mathrm{d} t}=\dot{D}_{\mathrm{FP}}\left(v-v_{\mathrm{FP}}\right)+D_{\mathrm{FP}}^{\prime}\left(r-r_{\mathrm{FP}}\right)
\end{array} .\right.
$$

Here a "prime" denotes differentiation respect to $r$, while a "dot" denotes differentiation respect to $v$, and the subscript FP means that the derivatives are evaluated at the fixed point (singularity). In order to characterize the singularity we have to study the eigenvalues of the stability matrix $J$ of the system (30)

$$
\chi_{ \pm}=\frac{1}{2}\left(\operatorname{Tr} J \pm \sqrt{(\operatorname{Tr} J)^{2}-4 \operatorname{det} J}\right)
$$

where

$$
\begin{aligned}
& \operatorname{Tr} J=\dot{N}_{\mathrm{FP}}+D_{\mathrm{FP}}^{\prime}=1-2 M_{F P}^{\prime} \\
& \operatorname{det} J=\dot{N}_{\mathrm{FP}} D_{\mathrm{FP}}^{\prime}-\dot{D}_{\mathrm{FP}} N_{\mathrm{FP}}^{\prime}=4 \dot{M}_{F P} .
\end{aligned}
$$


A singularity is called locally naked if the FP is a repulsive node $\left(\operatorname{Tr} J>0\right.$, $\left.\operatorname{det} J>0, \operatorname{and}(\operatorname{Tr} J)^{2}-4 \operatorname{det} J>0\right)$. The strength of the singularity is given by

$$
S=\frac{\dot{M}_{F P} X_{F P}^{2}}{2}
$$

where $X_{F P} \equiv \lim _{(r, v) \rightarrow \mathrm{FP}} \frac{v(r)}{r}$ is the tangent vector to the radial null geodesic at the FP. The singularity is strong if $S>0$, otherwise it is called integrable. The tangent vectors $X_{F P}$ defining the directions of the trajectories near the singularity are the non-marginal characteristic directions relative to the eigenvalues $\chi_{ \pm}$. One sees that these characteristic directions are given by

$$
r(v)=r_{F P}+\frac{\chi_{ \pm}}{2}\left(v-v_{F P}\right)
$$

so that $X_{F P}=\frac{2}{\chi_{ \pm}}$. In particular if $\operatorname{det} J \neq 0$ one obtains the expression $X_{F P}=\frac{2 \chi_{\mp}}{\operatorname{det} J}$ (the same relation found by 32 with a different method). As an example, we first review the simple Vaidya case, in which the generalized mass function is $M_{0}(r, v)=m(v) G_{0}$. In this case the singularity is the point $\mathrm{FP}=(0,0)$, and:

$$
\operatorname{Tr} J=1-2 M_{0}^{\prime}=1 \quad \operatorname{det} J=4 \dot{M}_{0}=4 \lambda G_{0}
$$

thus

$$
\chi_{ \pm}=\frac{1}{2}\left(1 \pm \sqrt{1-16 \lambda G_{0}}\right)
$$

and the point $(0,0)$ is a locally naked singularity if $\lambda \leq \frac{1}{16 G_{0}}$. It should be noticed that the linear solutions $r(v)=$ $\frac{\chi_{ \pm}}{2} v \equiv \mu_{ \pm} v$ are the characteristic directions of the corresponding dynamical system's fixed point. In particular in this case $X_{F P}=\frac{\mu_{\mp}}{\lambda G_{0}}$ and therefore $r=0$ is strong singularity $\left(\dot{M}_{0}>0\right)$.

In the improved Vaidya case the generalized mass function is $M_{k}(r, v)=m(v) G(r)$ with $\lim _{r \rightarrow 0} G(r)=0$, and the fixed points are the solutions of the following system

$$
\left\{\begin{array}{l}
2 r=0 \\
r-\frac{2 \lambda v G_{0}}{r+\alpha \sqrt{\lambda}} r=0
\end{array}\right.
$$

Therefore a line of fixed points is present at $r=0$ and for a given fixed point of the type $\left(0, v_{0}\right)$

$$
\operatorname{Tr} J=1-2 M_{F P}^{\prime}=1-\frac{2 \lambda v_{0} G_{0}}{\alpha \sqrt{\lambda}}, \quad \operatorname{det} J=4 \dot{M}_{F P}=0 .
$$

In particular, the determinant is zero because the running gravitational coupling $G(r)$ is zero in the limit $r \rightarrow 0$. This fact implies that one of the eingenvalues of $J$ is always $\chi_{-}=0$, while the other one depends on the position of the fixed point in the line $r=0$

$$
\chi_{+}\left(v_{0}\right) \equiv \operatorname{Tr} J=1-\frac{2 \lambda v_{0} G_{0}}{\alpha \sqrt{\lambda}} .
$$

In this case the singularities form a line of improper nodes (in the dynamical system classification). The characteristic directions associated with the lines are

$$
r=0 \quad v=v_{0}+\frac{2 r}{\chi_{+}\left(v_{0}\right)}
$$

The only non-marginal direction is the family $X_{F P}=\frac{2}{\chi_{+}\left(v_{0}\right)}$ and (assuming that we can compute the strength of the singularity as in the case of a node) because of $\operatorname{det} J=4 \dot{M}_{F P}=0$, in our case the strength of the singularity is zero. The improved Vaidya space-time is thus characterized by a line of integrable singularities at $r=0$.

We have to remark that this result does not depend on the cutoff identification. The strength of the singularity is zero as the Newton's constant tends to zero in the ultraviolet limit $k \rightarrow \infty$, i.e. for $r \rightarrow 0$, and thus this is a general result of Asymptotic Safety. 
This result can also be understood in a more intuitive way: for a given dynamical system of the type $\dot{\mathbf{x}}(t)=\mathbf{f}[\mathbf{x}(t)]$, the volume $V(t)$ of a cluster of initial conditions in the phase space $\{\mathbf{x}(t)\}$ evolves according to

$$
\frac{1}{V(t)} \frac{\mathrm{d} V(t)}{\mathrm{d} t}=\nabla \cdot \mathbf{f}[\mathbf{x}(t)] \equiv \operatorname{det} J
$$

Therefore, if $\nabla \cdot \mathbf{f} \neq 0 \forall t$ then $V(t) \rightarrow 0$ for $t \rightarrow+\infty$ (or $t \rightarrow-\infty$, depending on the positivity of $\operatorname{Tr} J$ ), i.e. the trajectories will asymptotically approch a geometrical object having zero-measure, as it happens in the classical VKP model. In our case $\nabla \cdot \mathbf{f}=0 \forall t$, thus the volume of a cluster of initial conditions is always conserved: this fact implies that an object falling into the singularity is not "destroyed" by the tidal forces (its volume is not reduced to zero) and the singularity is integrable.

\section{CONCLUSIONS}

In this paper we have presented a dynamical model of a gravitational collapse based on a RG improved Vaidya metric by using the RG equation for QEG. Our conclusions are that for a generic set of initial conditions the singularity is globally naked, even for flow conditions that were not naked in the classical case. On the other hand, due to the running of the gravitational coupling at Planck energies, the divergence of the Ricci curvature is much milder than in the classical case. In particular the singularity is always integrable for any value of the efficiency of the accrection mass scale $\lambda$. It must be stressed that inclusion of a possible running of the cosmological constant in the lapse function would not change our conclusions as this contribution, with the dynamical cutoff identification proposed in this paper, is always vanishing in the $r \rightarrow 0$ limit (as long as for $k^{2 a} \sim \rho$ with $a>1$ ). It would be interesting to discuss the possible astrophysical consequences of the presented model, an issue we plan to discuss in a separate publication.

\section{ACKNOWLEDGEMENTS}

A.P and B.K. acknowledge the support of FONDECYT 1120360 and 1161150.

[1] J. B. Hartle and S. W. Hawking, Phys. Rev. D 28, 2960 (1983)

[2] A. Vilenkin, Phys. Rev. D 37, 888 (1988)

[3] V. P. Frolov, M. A. Markov, and V. F. Mukhanov, Physics Letters B 216, 272 (1989).

[4] I. Dymnikova, General Relativity and Gravitation 24, 235 (1992).

[5] A. Bonanno and M. Reuter, Phys. Rev. D 62, 043008 (2000), hep-th/0002196

[6] B. Koch and F. Saueressig, Classical and Quantum Gravity 31, 015006 (2014), arXiv:1306.1546 [hep-th]

[7] C. Rovelli and F. Vidotto, International Journal of Modern Physics D 23, 1442026 (2014), arXiv:1401.6562 [gr-qc]

[8] T. De Lorenzo, C. Pacilio, C. Rovelli, and S. Speziale, General Relativity and Gravitation 47, 41 (2015), arXiv:1412.6015 $[\mathrm{gr}-\mathrm{qc}]$.

[9] C. Gundlach, R. H. Price, and J. Pullin, Phys. Rev. D 49, 883 (1994), gr-qc/9307009.

[10] E. Poisson and W. Israel, Classical and Quantum Gravity 5, L201 (1988)

[11] O. Lauscher and M. Reuter, Phys. Rev. D 66, 025026 (2002), hep-th/0205062

[12] M. Niedermaier and M. Reuter, Living Reviews in Relativity 9 (2006), 10.12942/lrr-2006-5.

[13] A. Codello and R. Percacci, Physical Review Letters 97, 221301 (2006), hep-th/0607128.

[14] M. Reuter, Phys. Rev. D 57, 971 (1998), hep-th/9605030

[15] A. Bonanno and M. Reuter, Journal of High Energy Physics 2, 035 (2005), hep-th/0410191

[16] M. Reuter and F. Saueressig, Phys. Rev. D 65, 065016 (2002), hep-th/0110054

[17] A. Nink and M. Reuter, Journal of High Energy Physics 1, 62 (2013), arXiv:1208.0031 [hep-th]

[18] A. Bonanno and M. Reuter, Phys. Rev. D 65, 043508 (2002), hep-th/0106133

[19] M. Reuter and F. Saueressig, JCAP 9, 012 (2005), hep-th/0507167

[20] A. Bonanno, G. Esposito, C. Rubano, and P. Scudellaro, Classical and Quantum Gravity 23, 3103 (2006), astro$\mathrm{ph} / 0507670$

[21] A. Bonanno and M. Reuter, JCAP 8, 024 (2007), arXiv:0706.0174 [hep-th]

[22] G. D'Odorico and F. Saueressig, Phys. Rev. D 92, 124068 (2015).

[23] R. Torres, Physics Letters B 733, 21 (2014) arXiv:1404.7655 [gr-qc],

[24] R. Torres and F. Fayos, Physics Letters B 733, 169 (2014), arXiv:1405.7922 [gr-qc],

[25] Y. Kuroda, Progress of Theoretical Physics 72, 63 (1984). 
[26] A. Papapetrou, in A Random Walk in Relativity and Cosmology, edited by M. Dadhich, J. Krishna Rao, J. V. Narlikar, and C. V. Vishveshwara (1985) pp. 184-191.

[27] F. J. Tipler, Phys. Rev. D 15, 942 (1977)

[28] M. Reuter, Phys. Rev. D 57, 971 (1998), hep-th/9605030

[29] S.-B. Liao and M. Strickland, Phys. Rev. D 52, 3653 (1995), hep-th/9501137

[30] S.-B. Liao and M. Strickland, Nuclear Physics B 532, 753 (1998), hep-th/9803173

[31] L. P. Hughston, International Journal of Theoretical Physics 4, 267 (1971)

[32] M. D. Mkenyeleye, R. Goswami, and S. D. Maharaj, Phys. Rev. D 90, 064034 (2014)

[33] P. S. Joshi, Oxford UP (International series of monographs on physics) 87 (1993).

[34] P. C. Vaidya, Physical Review 83, 10 (1951).

[35] P. C. Vaidya, Astrophys. J. 144, 943 (1966)

[36] P. Donà, A. Eichhorn, and R. Percacci, Phys. Rev. D 89, 084035 (2014), arXiv:1311.2898 [hep-th].

[37] I. H. Dwivedi and P. S. Joshi, Classical and Quantum Gravity 6, 1599 (1989)

[38] I. H. Dwivedi and P. S. Joshi, Classical and Quantum Gravity 8, 1339 (1991)

[39] W. Israel, Canadian Journal of Physics 63, 34 (1985) 